\title{
Anti-IgA antibodies in rheumatic and pulmonary disease
}

\author{
RONALD C. EGGERT,* DONNA J. BRAUER, $\dagger$ AND ERSKINE M. CAPERTON, JR. \\ Section of Arthritis, Department of Medicine, University of Minnesota Hospitals, \\ Minneapolis, Minnesota 55455, U.S.A.
}

Two observations underlie the hypothesis upon which this study was undertaken. The first is that IgA is the predominant class of immunoglobulins in bronchial secretions (Keimowitz, 1964). Externallysecreted $\operatorname{IgA}$ and, to a lesser extent, serum $\operatorname{IgA}$ participate in the immunological response to respiratory infection (Rossen, Butler, Waldman, Alford, Hornick, Togo, and Kasel, 1970; Smith, Bellanti, and Chanock, 1967) and it has been suggested that IgA may play a role in regulating the normal bacterial and viral flora of external membranes (Tomasi, 1968). The second observation is that one stimulus to rheumatoid factor production may be IgG antibody complexed to antigen (Henney, 1969). Rheumatoid factors occur in a variety of acute and chronic infections (Bartfeld, 1969) and their titres fall when the antigenic stimuli are removed (Williams and Kunkel, 1962). Thus, the relationship between IgA and pulmonary defence mechanisms combined with the knowledge that chronic antigenic stimulus may lead to production of anti-antibodies prompted speculation that, if a chronic antigenic stimulus is present in some pulmonary diseases, antibody against IgA might be found in patients with these diseases.

This study presents a survey of patients with chronic pulmonary disease and of control groups for the presence of anti-IgA antibodies. Patients were also studied for anti-IgG antibodies (rheumatoid factor) and cryoprecipitates. The results show that antibodies to $\operatorname{IgA}$ and cryoprecipitates occur with relative frequency in the sera of patients who have rheumatic or immunological disease when chronic pulmonary disease is associated. Anti-IgA antibodies do not occur in blood donors, other hospitalized patients (one exception noted below), patients with pulmonary disease not associated with underlying rheumatic or immunological disease, or patients with rheumatic disease but without demonstrable pulmonary disease.

\section{Methods}

MONOCLONAL PROTEIN PREPARATION

Monoclonal proteins were obtained from patients with multiple myeloma. Separation of $\operatorname{IgA}$ from the other immunoglobulins was facilitated by having a patient whose IgA peak migrated in the alpha $_{2}$ region on serum protein electrophoresis. Whole serum was subjected to starch block electrophoresis. The starch block eluates were vacuum concentrated, dialysed against $0.02 \mathrm{M}$ phosphate buffer at pH 8, and purified by DEAE-cellulose column chromatography. IgG was eluted from the column with $0.02 \mathrm{M}$ phosphate buffer at $\mathrm{pH} 8$ and $\mathrm{IgA}$ was then eluted with $0.15 \mathrm{M}$ phosphate buffer at $\mathrm{pH} 6.8$. Purity of the monoclonal proteins was demonstrated by immunoelectrophoresis against anti-whole serum and monospecific antisera (Hyland) and by radial immunodiffusion (Mancini, Carbonara, and Heremans, 1965).

\section{LATEX-FIXATION TEST}

This was performed by the method of Singer and Plotz (1956). Latex particles, mean diameter $0.81 \mu$, were employed (Kallestad). Whole serum was heat-inactivated at $56^{\circ} \mathrm{C}$. for $30 \mathrm{~min}$ and incubated with latex particles at $56^{\circ} \mathrm{C}$. for $2 \mathrm{hrs}$. Serial dilutions from $1: 5$ to $1: 5,120$ were tested. Agglutination of 2+ or greater in a dilution of $1: 40$ or greater was graded positive and 1+ agglutination or no agglutination was graded negative. Known positive and negative control sera were tested simultaneously with each group of test sera. Test sera did not agglutinate uncoated latex particles. Anti-IgG antibody activity was detected by coating the latex particles with commercially prepared human Fraction II (Hyland) in the screening studies and with Fraction II or monoclonal IgG in the adsorption and inhibition studies. Anti-IgA antibody activity was detected by coating latex particles with monoclonal IgA. Rabbit and goat anti-human-IgA anti-sera agglutinated latex particles coated with the monoclonal IgA and did not agglutinate uncoated, IgG-coated, or Fraction II-coated latex particles.

CR Y OPRE CIPITATES

Immediately after drawing, the blood sample was placed in an incubator at $37^{\circ} \mathrm{C}$. and allowed to clot for $2 \mathrm{hrs}$. The 
sample was then centrifuged at 2,000 r.p.m. for $10 \mathrm{~min}$. and the serum was separated immediately. Serum was allowed to stand at $4^{\circ} \mathrm{C}$. for $72 \mathrm{hrs}$, during which time it was observed at daily intervals for the presence of precipitation. Cryoprecipitates were separated by spinning sera at 15,000 r.p.m. in a Servall centrifuge at $4^{\circ} \mathrm{C}$. for $30 \mathrm{~min}$. and were purified by multiple re-suspensions at $37^{\circ} \mathrm{C}$. and re-precipitations at $4^{\circ} \mathrm{C}$. in phosphate-buffered saline $\mathrm{pH} 7 \cdot 4$. The purified cryoprecipitates were re-suspended in phosphate-buffered saline at $37^{\circ} \mathrm{C}$. and the immunoglobulin content was determined by immunoelectrophoresis against antiwhole serum and monospecific antisera. Antibody activity in the cryoprecipitates was determined by the latex test.

\section{IMMUNOADSORPTION}

Monoclonal IgG and IgA were insolubilized by crosslinkage to glutaraldehyde and antibody was adsorbed from the sera by the method of Avrameas and Ternynck (1969). The amount of insolubilized protein used as immunoadsorbant was $100 \mathrm{mg}$. and elution of adsorbed protein was accomplished with $0.1 \mathrm{M}$ glycine- $\mathrm{HCl}$ solution pH 2.8. Each serum $(0.8 \mathrm{ml}$.) was adsorbed with insolubilized IgG and IgA by this method six times. The unadsorbed serum and the adsorbed serum were then tested simultaneously for anti-IgA and anti-IgG activity by the latex-fixation test.

\section{INHIBITION STUDIES}

An inhibition experiment, also employing the latex test, was designed. Each test serum was diluted so that it contained enough antibody activity to give $2+$ agglutination of the latex particles at that and two higher dilutions, but not enough activity to agglutinate them at three higher dilutions. Diluted serum $(0 \cdot 125 \mathrm{ml}$.) was added to an equal volume of $0.1 \mathrm{M}$ glycine- $\mathrm{NaCl}$ solution $\mathrm{pH} 8 \cdot 2$ containing 0 , $\mathbf{0} \cdot 062,0 \cdot 125,0 \cdot 25$, or $0 \cdot 5 \mathrm{mg}$. inhibitor (FII, monoclonal IgG or monoclonal IgA). The serum with inhibitor was then added to $0.25 \mathrm{ml}$. latex suspension, the particles of which were coated with FII, monoclonal IgG, or monoclonal IgA. Two monoclonal IgG (one kappa type and one lambda type) and two monoclonal IgA (both kappa type, designated $K_{1}$ and $K_{2}$ ) proteins were employed.

\section{SEPARATION STUDIES}

For sucrose density gradient separations, sera were

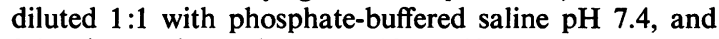
$0.3 \mathrm{ml}$. was layered over a 10 to 40 per cent. sucrose gradient and centrifuged at 35,000 r.p.m. for $18 \mathrm{hrs}$ in a Beckman model L-2 preparative ultra-centrifuge with an SL-50.1 rotor. Gradient fractions were collected from the bottom and assayed for total protein content. 19S and 7S fractions were pooled, and anti-IgA and anti-IgG activity determined by the latex fixation test.

Sephadex G-200 column separations were performed on $80 \times 1.5 \mathrm{~cm}$. columns equilibrated with $0.1 \mathrm{M}$ acetate buffer $\mathrm{pH} 3 \cdot 0$. Whole serum ( $2 \mathrm{ml}$.) dialysed to $\mathrm{pH} 3.0$ was employed. Each fraction was assayed for total protein content. Peak fractions were pooled and vacuum concentrated in collodion bags. Immunoglobulin content was determined by immunoelectrophoresis and anti-gammaglobulin activity was determined by the latex fixation test.

\section{OTHER PROCEDURES}

Serum immunoglobulin levels were measured by the radial immunodiffusion method (Mancini and others, 1965).
Haemolytic complement was determined in the immunochemistry laboratory of the University of Minnesota Hospitals by standard methods (Pickering, Gewurz, and Good, 1968).

\section{Subjects}

Three groups were studied:

(1) 57 patients who had infectious and non-infectious pulmonary diseases. In most of them the pulmonary disease was primary but in a few it was associated with rheumatic or immunological disease.

(2) 52 patients with rheumatic diseases, largely rheumatoid arthritis (R.A.) and systemic lupus erythematosus (S.L.E.), but without pulmonary involvement as determined by history, examination, chest roentgenograms and, in most cases, spirometry.

(3) 75 presumably healthy blood donors and 81 consecutively hospitalized patients.

Each patient with R.A. had a diagnosis of definite or classical disease by the criteria of the American Rheumatism Association (Committee of the A.R.A., 1964). Each patient with the diagnosis of S.L.E. had documented multisystem disease, a positive bead defibrination test for L.E. cells and anti-DNA antibody in the serum. A diagnosis of interstitial pulmonary disease (I.P.D.) wag based on history, examination, chest roentgenogrand pulmonary function studies, and autopsy (1 patient). A pulmonary function tests shown (Table II) were done aftep the patients had recovered from acute illness.

\section{Results}

\section{SCREENING STUDIES}

Eleven patients in Group 1 had anti-IgA antibody in their sera (Table I). The clinical diagnosis of these patients were: mixed cryoglobulinaemia with recurrent pulmonary infections (1); sicca syndrome with recurrent acute and chronic pulmonary infections (1); rheumatoid arthritis with I.P.D. and recurrent and/or chronic pulmonary infections (3); rheumatoid arthritis and I.P.D. (1); rheumatoid arthritis, chronic bronchitis, and bronchiectasis (1); chronic bronchitis and I.P.D. (1); mild polyarthritis with Raynaud's phenomenon and I.P.D. (1); pulmonary infiltrate with eosinophilia (P.I.E.) syndrome and I.P.D. (1); S.L.E. with recurrent pneumonia and possible pulmonary vasculitis (1).

Clinical diagnoses and pulmonary function studies are shown in Table II (overleaf). Each patient had a urine analysis, serum creatinine, and $24-\mathrm{hr}$ creatinine clearance; except in patients 1,2 , and 11 , the results were normal. (In Patient 1 renal biopsy showed acute and chronic glomerulitis with glomerular IgG, complement, and fibrin deposits. Patients 2 and 11 had presumptive glomerular disease on the basis of lowered serum complement and abnormal urine sediment). Each also had serum protein electrophoresis with normal or elevated alpha ${ }_{1}$ levels. 
Table I Subjects screened for anti-Ig $A$ and anti-IgG antibodies and cryoprecipitates

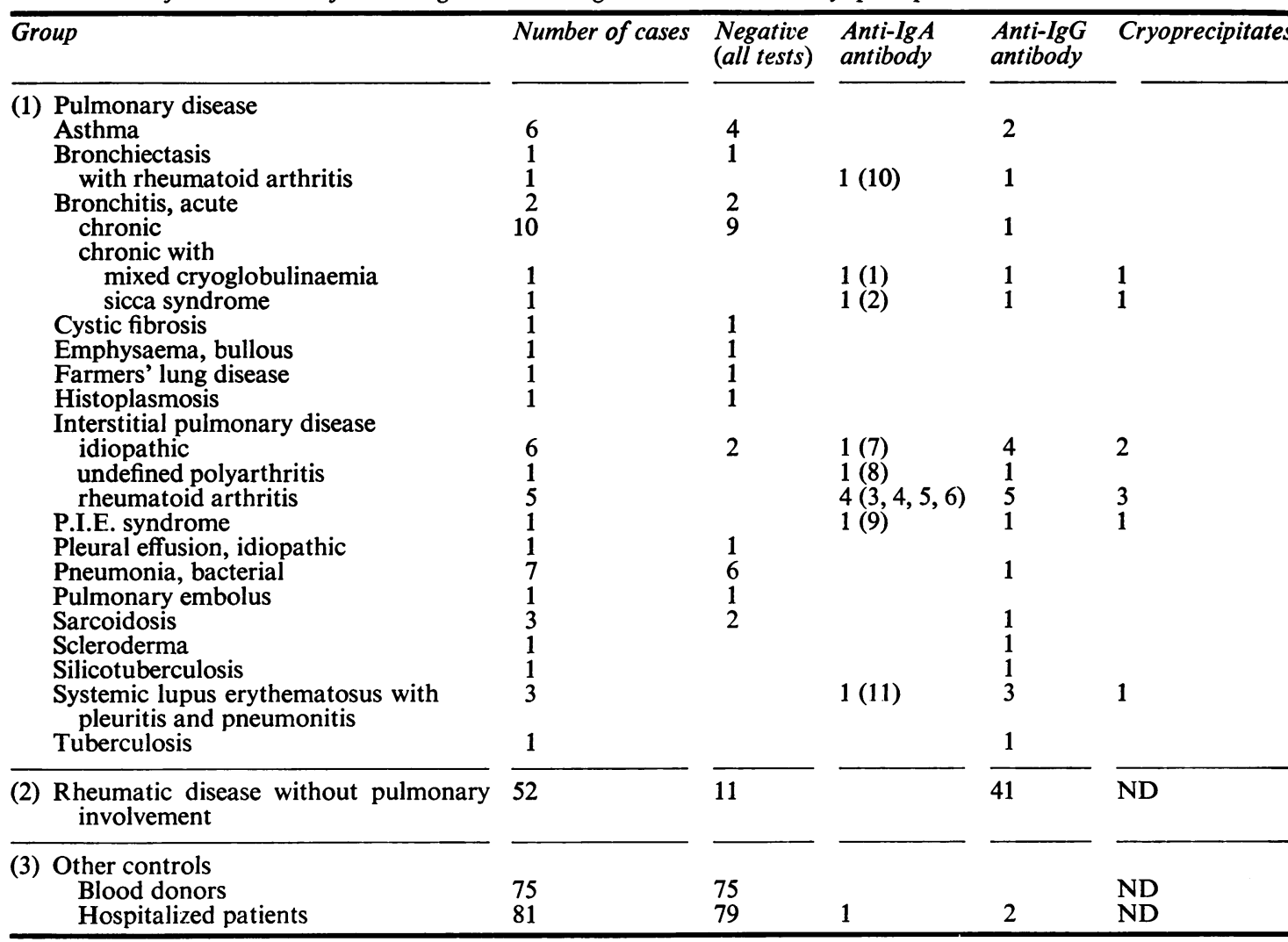

$\mathrm{ND}=$ not done Numerals in parentheses refer to patients discussed in text.

Table I also shows that there was one patient with rheumatoid arthritis and I.P.D., two with S.L.E. and pulmonary involvement, and five with idiopathic I.P.D. who had no anti-IgA antibody in their sera at the time of study. One hospitalized control patient had anti-IgA antibody. Her diagnosis was alcoholic cirrhosis with no pulmonary disease by history or demonstrable on chest roentgenogram. She had recently received multiple blood transfusions; because anti-IgA antibodies may develop following transfusion of incompatible IgA (Vyas, Holmdahl, Perkins, and Fudenberg, 1969), the relationship of the anti-IgA antibody to her underlying disease is uncertain. Two other patients with alcoholic cirrhosis were included among the hospitalized control patients and neither had anti-IgA antibody in his serum.

\section{SEROLOGICAL STUDIES}

Serological abnormalities in the eleven patients with pulmonary disease and anti-IgA antibody are shown in Table III (overleaf). Each patient with anti-IgA antibody also had anti-IgG antibody in his serum. The sera from eight of the eleven patients contained cryoprecipitates. All eight cryoprecipitates contained IgG and seven of the eight cryoprecipitates were mixtures of immunoglobulins. Four contained IgM and IgG; each of these had high-titre anti-IgG activity and no anti-IgA $(1,3$, and 11$)$ or low-titre anti-IgA (2) activity. Two cryoprecipitates contained IgG and IgA (4 and 9) and one contained IgM, IgG, and IgA (7); these cryoprecipitates had anti-IgG and anti-IgA activity. The eighth cryoprecipitate contained only IgG and had both anti-IgA and anti-IgG activity. Only one cryoprecipitate (Patient 1) contained a monoclonal protein: Monoclonal IgM with kappa-type light chains and polyclonal IgG. Other immunological data are shown in Table IV (overleaf).

\section{ADSORPTION STUDIES}

The results are shown in Table $\mathrm{V}$ (overleaf). A change in titre of one tube dilution was not considered significant because the serum was slightly diluted by a small amount of buffer remaining in the immunoadsorbant each time it was adsorbed. A change in titre of two tube dilutions or more was considered significant.

Adsorption of the test sera with insolubilized IgG reduced the anti-IgG titre by two tube dilutions in seven patients, three tube dilutions in three, and four tube dilutions in one. Adsorption with insolubilized 
Table II Clinical features of patients with anti-IgA antibody

\begin{tabular}{|c|c|c|c|c|c|c|c|c|c|c|c|}
\hline \multirow{2}{*}{$\begin{array}{l}\text { Patient } \\
\text { no. }\end{array}$} & \multirow{2}{*}{$\begin{array}{l}\text { Age } \\
(\text { yrs })\end{array}$} & \multirow[t]{2}{*}{$\operatorname{Sex}$} & \multirow[t]{2}{*}{ Systemic disease } & \multirow{2}{*}{$\begin{array}{l}\text { Clinical pulmonary } \\
\text { disease }\end{array}$} & \multirow[t]{2}{*}{ Chest $x$ ray } & \multicolumn{2}{|c|}{ Vital capacity } & \multicolumn{3}{|c|}{ Arterial blood } & \multirow{2}{*}{$\begin{array}{l}\text { Carbon } \\
\text { monoxide } \\
\text { diffusion } \\
\text { capacity }\end{array}$} \\
\hline & & & & & & $\begin{array}{l}1 \text { Second } \\
\text { (per cent. of } \\
\text { total) }\end{array}$ & $\begin{array}{l}\text { Total } \\
\text { (percent. of } \\
\text { predicted })\end{array}$ & pO2 & pCO2 & $p H$ & \\
\hline 1 & 34 & $\mathrm{~F}$ & $\begin{array}{l}\text { Mixed cryoglobulin- } \\
\text { aemia }\end{array}$ & $\begin{array}{l}\text { Recurrent pneumonia } \\
\text { Bronchitis } \\
\text { Sinusitis }\end{array}$ & Episodes of pneumonia & 44 & 54 & 74 & 28 & $7 \cdot 47$ & 22 \\
\hline 2 & 50 & $\mathrm{~F}$ & $\begin{array}{l}\text { Sicca syndrome with } \\
\text { renal failure }\end{array}$ & $\begin{array}{l}\text { Recurrent pneumonia } \\
\text { Chronic bronchitis }\end{array}$ & $\begin{array}{l}\text { Episodes of pneumonia } \\
\text { Recurrent pleural effu- } \\
\text { sions }\end{array}$ & 43 & 118 & 80 & 34 & $\overline{7 \cdot 41}$ & 27 \\
\hline 3 & 53 & $\mathrm{~F}$ & Rheumatoid arthritis & $\begin{array}{l}\text { Recurrent pneumonia } \\
\text { Chronic bronchitis }\end{array}$ & $\begin{array}{l}\text { Episodes of pneumonia } \\
\text { Linear interstitial infil- } \\
\text { trates }\end{array}$ & 50 & 35 & 48 & 36 & $7 \cdot 48$ & ND \\
\hline 4 & 58 & $\mathbf{M}$ & Rheumatoid arthritis & Chronic bronchitis & $\begin{array}{l}\text { Diffuse nodular inter- } \\
\text { stitial infiltrates }\end{array}$ & 38 & 78 & 78 & 32 & $7 \cdot 48$ & ND \\
\hline 5 & 57 & $\mathbf{F}$ & $\begin{array}{l}\text { Rheumatoid arthritis } \\
\text { Felty's syndrome }\end{array}$ & Asymptomatic & $\begin{array}{l}\text { Diffuse linear inter- } \\
\text { stitial infiltrates }\end{array}$ & 73 & 97 & 71 & 34 & $7 \cdot 49$ & 17 \\
\hline 6 & 33 & $\mathbf{F}$ & Rheumatoid arthritis & Recurrent pneumonia & $\begin{array}{l}\text { Episodes of pneumonia } \\
\text { Diffuse linear intersti- } \\
\text { tial infiltrates }\end{array}$ & ND & ND & ND & ND & ND & ND \\
\hline 7 & 55 & $\mathbf{F}$ & None & $\begin{array}{l}\text { Recurrent broncho- } \\
\text { pneumonia } \\
\text { Chronic bronchitis }\end{array}$ & $\begin{array}{l}\text { Dense nodular intersti- } \\
\text { tial infiltrates }\end{array}$ & 85 & 62 & 47 & 36 & $7 \cdot 54$ & ND \\
\hline 8 & 53 & $\mathbf{F}$ & $\begin{array}{l}\text { Polyarthritis } \\
\text { Raynaud's phenom- } \\
\text { enon }\end{array}$ & $\begin{array}{l}\text { Pneumonia } \\
\text { Recurrent acute bron- } \\
\text { chitis } \\
\text { Autopsy-proven inter- } \\
\text { stitial pulmonary } \\
\text { fibrosis }\end{array}$ & $\begin{array}{l}\text { Dense nodular and } \\
\text { linear interstitial in- } \\
\text { filtrates }\end{array}$ & 85 & 62 & 59 & 39 & $7 \cdot 44$ & $10 \cdot 5$ \\
\hline 9 & 47 & $\mathbf{F}$ & Multiple sclerosis & $\begin{array}{l}\text { Pulmonary infiltrate } \\
\text { with eosinophilia } \\
\text { syndrome }\end{array}$ & $\begin{array}{l}\text { Extensive alveolar in- } \\
\text { filtrates } \\
\text { Linear interstitial in- } \\
\text { filtrates after treat- } \\
\text { ment }\end{array}$ & 66 & 91 & 81 & 32 & $7 \cdot 45$ & 21 \\
\hline 10 & 19 & $\mathbf{F}$ & Rheumatoid arthritis & $\begin{array}{l}\text { Recurrent pneumonia } \\
\text { Chronic bronchitis }\end{array}$ & $\begin{array}{l}\text { Bronchiectasis } \\
\text { (bronchogram) }\end{array}$ & 79 & 62 & 73 & 37 & $7 \cdot 41$ & ND \\
\hline 11 & 16 & $\mathbf{M}$ & $\begin{array}{l}\text { Systemic lupus ery- } \\
\text { thematosus }\end{array}$ & $\begin{array}{l}\text { Pleuritis } \\
\text { Recurrent pneumonia }\end{array}$ & Episodes of pneumonia & ND & ND & ND & ND & ND & ND \\
\hline
\end{tabular}

* Normal Dco (Single breath technique): 25-30 ml. CO/min./mm. Hg.

ND $=$ not done.

Table III Serological abnormalities

\begin{tabular}{|c|c|c|c|c|c|c|c|}
\hline \multirow[t]{3}{*}{ Patient no. } & \multirow{2}{*}{\multicolumn{2}{|c|}{$\frac{\text { Serum }}{\text { Latex }}$}} & \multicolumn{5}{|c|}{ Cryoprecipitate } \\
\hline & & & \multicolumn{2}{|l|}{ Latex } & \multicolumn{3}{|c|}{ Immunoelectrophoresis } \\
\hline & Anti-IgA & Anti-FII & Anti-IgA & Anti-FII & $\operatorname{Ig} M$ & $\operatorname{Ig} G$ & $\operatorname{Ig} A$ \\
\hline 2 & $1: 160$ & $1: 320$ & $1: 20$ & $1: 1,280$ & + & + & - \\
\hline 1 & $1: 320$ & $1: 1,280$ & & $1: 80$ & + & + & - \\
\hline 3 & $1: 640$ & $1: 1,280$ & 0 & $1: 160$ & + & + & - \\
\hline 11 & $1: 80$ & $1: 80$ & 0 & $1: 320$ & + & + & - \\
\hline 4 & $1: 160$ & $1: 1,280$ & $1: 20$ & $1: 80$ & - & + & + \\
\hline 9 & $1: 320$ & $1: 80$ & $1: 160$ & $1: 640$ & - & + & + \\
\hline 7 & $1: 80$ & $1: 80$ & $1: 80$ & $1: 80$ & + & + & + \\
\hline 6 & $1: 1,280$ & $1: 2,560$ & $1: 40$ & $1: 640$ & - & + & - \\
\hline 8 & $1: 80$ & $1: 80$ & & & & & \\
\hline 10 & $1: 640$ & $1: 1,280$ & & & & & \\
\hline 5 & $1: 320$ & $1: 640$ & & & & & \\
\hline
\end{tabular}

IgG did not reduce the anti-IgA titre in five patients and reduced it by one tube dilution in six.

Conversely, adsorption with insolubilized IgA reduced the anti-IgA titre by two tube dilutions in six patients, three tube dilutions in three, and four tube dilutions in two. Adsorption with insolubilized IgA caused no change in the anti-IgG titre in six patients and a change of one tube dilution in five.
INHIBITION ST UDIES

The results are shown in Table VI (overleaf). Inhibitor concentration of $0.062 \mathrm{mg}$. did not inhibit latex agglutination in any experiment and these results are therefore omitted from the Table.

In six patients $(4,8,2,1,5$, and 6$)$, agglutination of FII-or IgG-coated latex particles was inhibited by FII and/or IgG but not by IgA. Similarly in these 
Table IV Immunological studies

\begin{tabular}{|c|c|c|c|c|c|c|}
\hline \multirow[t]{2}{*}{ Patient no. } & \multirow[t]{2}{*}{ L.E. cells } & \multirow{2}{*}{$\begin{array}{l}\text { Anti-DNA } \\
\text { antibody }\end{array}$} & \multirow{2}{*}{$\begin{array}{l}\text { Haemolytic } \\
\text { complement }\end{array}$} & \multicolumn{3}{|c|}{ Serum immunoglobulin levels } \\
\hline & & & & $I g M$ & $I g G$ & $\operatorname{Ig} A$ \\
\hline $\begin{array}{r}1 \\
2 \\
3 \\
4 \\
5 \\
6 \\
7 \\
8 \\
9 \\
10 \\
11\end{array}$ & $\begin{array}{l}- \\
\overline{+} \\
+ \\
\text { ND } \\
- \\
\bar{N} \\
- \\
- \\
- \\
+\end{array}$ & $\begin{array}{l}- \\
\overline{+} \\
\overline{-} \\
+ \\
- \\
- \\
- \\
- \\
- \\
+\end{array}$ & $\begin{array}{c}15-36 \\
44-55 \\
54 \\
89 \\
55 \\
76 \\
89 \\
112 \\
63 \\
52 \\
25\end{array}$ & $\begin{array}{l}130 \\
720 \\
640 \\
172 \\
425 \\
470 \\
213 \\
435 \\
172 \\
172 \\
232\end{array}$ & $\begin{array}{l}3,480 \\
1,400 \\
3,550 \\
1,900 \\
2,120 \\
1,370 \\
2,520 \\
4,641 \\
2,460 \\
1,630 \\
2,475\end{array}$ & $\begin{array}{r}560 \\
750 \\
455 \\
1,200 \\
400 \\
680 \\
1,000 \\
162 \\
630 \\
680 \\
266\end{array}$ \\
\hline
\end{tabular}

Normal values: Anti-DNA antibody, negative; haemolytic complement, 47-70 C'H50 units/ml.; IgM, 47-147 mg. per cent; IgG, 569-1919; IgA, 61-330.

$\mathrm{ND}=$ not done.

Table V Results of adsorption experiments

\begin{tabular}{|c|c|c|c|c|c|c|c|}
\hline \multirow[t]{2}{*}{ Patient no. } & \multirow{2}{*}{ Latex coat } & \multicolumn{3}{|c|}{ Adsorption with $\operatorname{IgG}$} & \multicolumn{3}{|c|}{ Adsorption with $\operatorname{Ig} A$} \\
\hline & & Titre before & Titre after & $\begin{array}{l}\text { Change titre } \\
\text { (tube dilutions) }\end{array}$ & Titre before & Titre after & $\begin{array}{l}\text { Change titre } \\
\text { (tube dilutions) }\end{array}$ \\
\hline \multirow[t]{2}{*}{10} & IgG & $1: 640$ & $1: 80$ & 3 & $1: 640$ & $1: 320$ & 1 \\
\hline & IgA & $1: 320$ & $1: 320$ & 0 & $1: 320$ & $1: 20$ & 4 \\
\hline \multirow[t]{2}{*}{4} & IgG & $1: 1,280$ & $1: 80$ & 4 & $1: 1,280$ & $1: 1,280$ & 0 \\
\hline & IgA & $1: 160$ & $1: 80$ & 1 & $1: 160$ & $1: 40$ & 2 \\
\hline \multirow[t]{2}{*}{5} & IgG & $1: 1,280$ & $1: 320$ & 2 & $1: 1,280$ & $1: 1,280$ & 0 \\
\hline & IgA & $1: 640$ & $1: 320$ & $\overline{1}$ & $1: 640$ & $1: 40$ & 4 \\
\hline \multirow[t]{2}{*}{7} & IgG & $1: 80$ & $1: 10$ & 3 & $1: 80$ & $1: 80$ & 0 \\
\hline & IgA & $1: 80$ & $1: 40$ & 1 & $1: 80$ & $1: 10$ & 3 \\
\hline \multirow[t]{2}{*}{6} & IgG & $1: 2,560$ & $1: 640$ & 2 & $1: 2,560$ & $1: 1,280$ & 1 \\
\hline & IgA & $1: 1,280$ & $1: 1,280$ & 0 & $1: 1,280$ & $1: 160$ & 3 \\
\hline \multirow[t]{2}{*}{3} & IgG & $1: 1,280$ & $1: 320$ & 2 & $1: 1,280$ & $1: 1,280$ & 0 \\
\hline & IgA & $1: 640$ & $1: 320$ & 1 & $1: 640$ & $1: 80$ & 3 \\
\hline \multirow{2}{*}{11} & IgG & $1: 80$ & $1: 10$ & 3 & $1: 80$ & $1: 40$ & 1 \\
\hline & IgA & $1: 80$ & $1: 80$ & 0 & $1: 80$ & $1: 20$ & 2 \\
\hline \multirow{2}{*}{9} & IgG & $1: 80$ & $1: 20$ & 2 & $1: 80$ & $1: 80$ & 0 \\
\hline & IgA & $1: 320$ & $1: 160$ & $\overline{1}$ & $1: 320$ & $1: 80$ & 2 \\
\hline \multirow[t]{2}{*}{8} & IgG & $1: 80$ & $1: 20$ & 2 & $1: 80$ & $1: 40$ & 1 \\
\hline & IgA & $1: 80$ & $1: 80$ & 0 & $1: 80$ & $1: 20$ & 2 \\
\hline \multirow[t]{2}{*}{2} & IgG & $1: 320$ & $1: 80$ & 2 & $1: 320$ & $1: 160$ & $\overline{1}$ \\
\hline & IgA & $1: 160$ & $1: 160$ & 0 & $1: 160$ & $1: 40$ & 2 \\
\hline \multirow{2}{*}{1} & IgG & $1: 1,280$ & $1: 320$ & 2 & $1: 1,280$ & $1: 1,280$ & 0 \\
\hline & IgA & $1: 320$ & $1: 160$ & 1 & $1: 320$ & $1: 80$ & 2 \\
\hline
\end{tabular}

patients agglutination of IgA-coated latex particles was inhibited by IgA but not by FII or IgG. In three patients $(9,7$, and 3$)$, inhibition overlap occurred at the highest inhibitor concentration. In Patient 9, IgG (K) and IgG (lambda) inhibited agglutination of IgA $\left(K_{2}\right)$ at $0.5 \mathrm{mg}$. inhibitor level; however, only IgA inhibited agglutination at the two lower concentrations and in the two experiments with FII- and IgG-coated latex particles IgA did not inhibit agglutination at any concentration. Similar results obtained in patients 7 and 3. For patient 10, IgG, but not IgA, inhibited IgG-coated latex agglutination but both IgG and IgA inhibited agglutination of IgAcoated latex particles with a separation of only one inhibitor concentration.
SEPARATION ST UDIES

The results are shown in Table VII (overleaf). AntiIgG activity was demonstrated in the $19 \mathrm{~S}$ fraction by sucrose density gradient separation in three patients $(7,10$, and 6) and in both $19 S$ and $7 \mathrm{~S}$ fractions in two (9 and 5). Anti-IgA activity was found in the 19S fraction in one patient (7) and in the 7S fraction in the others. Sephadex G-200 column separations confirmed the sucrose density gradient results, except in Patient 5, where no anti-gammaglobulin activity could be demonstrated in peak II despite multiple separations.

Discussion

Eleven patients with chronic pulmonary disease have 
Table VI Results of inhibition experiments

\begin{tabular}{|c|c|c|c|c|c|c|}
\hline \multicolumn{2}{|c|}{$\begin{array}{l}\text { Patient Latex coat } \\
\text { no. }\end{array}$} & \multicolumn{4}{|c|}{$\begin{array}{l}\text { Inhibitor } \\
\text { concentration (mg.) }\end{array}$} & \multirow[t]{2}{*}{ Inhibitor } \\
\hline & & 0 & $0 \cdot 125$ & 0.25 & 0.5 & \\
\hline \multirow[t]{2}{*}{4} & FII & $\begin{array}{l}+ \\
+ \\
+ \\
+ \\
+\end{array}$ & $\begin{array}{l}+ \\
+ \\
+ \\
+ \\
+\end{array}$ & $\begin{array}{l}- \\
- \\
-- \\
+ \\
+\end{array}$ & $\begin{array}{l}- \\
- \\
- \\
+ \\
+\end{array}$ & $\begin{array}{l}\text { FII } \\
\text { IgG(K) } \\
\text { IgG (lambda) } \\
\operatorname{IgA}\left(K_{1}\right) \\
\operatorname{IgA}\left(K_{2}\right)\end{array}$ \\
\hline & $\operatorname{IgA}\left(\mathrm{K}_{1}\right)$ & $\begin{array}{l}+ \\
+ \\
+ \\
+ \\
+\end{array}$ & $\begin{array}{l}+ \\
+ \\
+ \\
+\end{array}$ & $\begin{array}{l}+ \\
+ \\
+ \\
- \\
-\end{array}$ & $\begin{array}{l}+ \\
+ \\
+ \\
- \\
-\end{array}$ & $\begin{array}{l}\text { FII } \\
\text { IgG(K) } \\
\text { IgG (lambda) } \\
\operatorname{IgA}\left(K_{1}\right) \\
\operatorname{IgA}\left(K_{2}\right)\end{array}$ \\
\hline \multirow[t]{2}{*}{8} & $\operatorname{IgG}(\mathrm{K})$ & + & $\begin{array}{l}+ \\
+ \\
+ \\
+\end{array}$ & $\begin{array}{l}+ \\
- \\
+ \\
+\end{array}$ & $\begin{array}{l}- \\
- \\
+ \\
+\end{array}$ & $\begin{array}{l}\text { FII } \\
\operatorname{IgG}(K) \\
\operatorname{IgA}\left(K_{1}\right) \\
\operatorname{IgA}\left(K_{2}\right)\end{array}$ \\
\hline & $\operatorname{IgA}\left(\mathrm{K}_{1}\right)$ & + & $\begin{array}{l}+ \\
+ \\
+ \\
+ \\
+\end{array}$ & $\begin{array}{l}+ \\
+ \\
+ \\
- \\
-\end{array}$ & $\begin{array}{l}+ \\
+ \\
+ \\
- \\
-\end{array}$ & $\begin{array}{l}\text { FII } \\
\operatorname{IgG}(\mathrm{K}) \\
\operatorname{IgG}(\operatorname{lambda}) \\
\operatorname{IgA}\left(\mathrm{K}_{1}\right) \\
\operatorname{IgA}\left(\mathrm{K}_{2}\right)\end{array}$ \\
\hline \multirow[t]{2}{*}{2} & $\operatorname{IgG}(\mathrm{K})$ & $t^{\prime}$ & $\begin{array}{l}+ \\
+ \\
+\end{array}$ & $\begin{array}{l}+ \\
\overline{+} \\
+\end{array}$ & $\begin{array}{l}+ \\
+ \\
+\end{array}$ & $\begin{array}{l}\text { FII } \\
\operatorname{IgG}(K) \\
\operatorname{IgA}\left(K_{2}\right)\end{array}$ \\
\hline & $\operatorname{IgA}\left(\mathrm{K}_{2}\right)$ & $\top$ & $\begin{array}{l}+ \\
+ \\
-\end{array}$ & $\begin{array}{l}+ \\
+ \\
-\end{array}$ & $\begin{array}{l}+ \\
+ \\
-\end{array}$ & $\begin{array}{l}\text { FII } \\
\operatorname{IgG}(K) \\
\operatorname{IgA}\left(K_{2}\right)\end{array}$ \\
\hline \multirow[t]{2}{*}{1} & $\operatorname{IgG}(\mathrm{K})$ & + & $\begin{array}{l}+ \\
+ \\
+ \\
+\end{array}$ & $\begin{array}{l}+ \\
- \\
+ \\
+\end{array}$ & $\begin{array}{l}+ \\
- \\
+ \\
+\end{array}$ & $\begin{array}{l}\text { FII } \\
\text { IgG(K) } \\
\text { IgG (lambda) } \\
\operatorname{IgA}\left(K_{2}\right)\end{array}$ \\
\hline & $\operatorname{IgA}\left(\mathrm{K}_{2}\right)$ & & $\begin{array}{l}+ \\
+ \\
+ \\
+\end{array}$ & $\begin{array}{l}+ \\
+ \\
+ \\
-\end{array}$ & $\begin{array}{l}+ \\
+ \\
+ \\
-\end{array}$ & $\begin{array}{l}\text { FII } \\
\operatorname{IgG}(\mathrm{K}) \\
\operatorname{IgG}(\text { lambda) } \\
\operatorname{IgA}\left(\mathrm{K}_{2}\right)\end{array}$ \\
\hline \multirow[t]{2}{*}{5} & $\operatorname{IgG}(\mathrm{K})$ & + & $\begin{array}{l}+ \\
+ \\
+\end{array}$ & $\begin{array}{l}+ \\
- \\
+\end{array}$ & $\begin{array}{l}- \\
+\end{array}$ & $\begin{array}{l}\text { FII } \\
\operatorname{IgG}(K) \\
\operatorname{IgA}\left(K_{2}\right)\end{array}$ \\
\hline & $\operatorname{IgA}\left(\mathrm{K}_{2}\right)$ & + & $\begin{array}{l}+ \\
+ \\
+\end{array}$ & $\begin{array}{l}+ \\
+ \\
-\end{array}$ & $\begin{array}{l}+ \\
+ \\
-\end{array}$ & $\begin{array}{l}\text { FII } \\
\operatorname{IgA}\left(K_{1}\right) \\
\operatorname{IgA}\left(K_{2}\right)\end{array}$ \\
\hline
\end{tabular}

been found to have antibodies to whole human $\operatorname{IgA}$ present in their sera. Nine of these patients had definite associated rheumatic or immunological disease: mixed cryoglobulinaemia, rheumatoid arthritis, undefined polyarthritis, S.L.E., and the sicca syndrome. In addition, the P.I.E. syndrome (1 patient) may be a manifestation of an underlying immunological disorder (Hall, Kozak, and Spink, 1964). One patient (No. 7) had chronic bronchitis and I.P.D.; this was the only patient in the group who had no definite or possible associated rheumatic or immunological disease.

Insofar as may be ascertained by clinical features,

\begin{tabular}{|c|c|c|c|c|c|c|}
\hline \multicolumn{2}{|c|}{$\begin{array}{l}\text { Patient Latex coat } \\
\text { no. }\end{array}$} & \multicolumn{4}{|c|}{$\begin{array}{l}\text { Inhibitor } \\
\text { concentration (mg.) }\end{array}$} & \multirow[t]{2}{*}{ Inhibitor } \\
\hline & & 0 & $0 \cdot 125$ & $0 \cdot 25$ & 0.5 & \\
\hline \multirow[t]{2}{*}{6} & $\operatorname{IgG}(\mathrm{K})$ & $\begin{array}{l}+ \\
+ \\
+ \\
+\end{array}$ & $\begin{array}{l}+ \\
+ \\
+ \\
+\end{array}$ & $\begin{array}{l}+ \\
- \\
+ \\
+\end{array}$ & $\begin{array}{l}+ \\
- \\
+ \\
+\end{array}$ & $\begin{array}{l}\text { FII } \\
\text { IgG(K) } \\
\text { IgG (lambda) } \\
\operatorname{IgA}\left(\mathrm{K}_{2}\right)\end{array}$ \\
\hline & $\operatorname{Ig} \mathrm{A}\left(\mathrm{K}_{2}\right)$ & $\begin{array}{l}+ \\
+ \\
+ \\
+\end{array}$ & $\begin{array}{l}+ \\
+ \\
+ \\
+\end{array}$ & $\begin{array}{l}+ \\
+ \\
+ \\
-\end{array}$ & $\begin{array}{l}+ \\
+ \\
+ \\
-\end{array}$ & $\begin{array}{l}\text { FII } \\
\text { IgG(K) } \\
\text { IgG (lambda) } \\
\operatorname{IgA}\left(\mathrm{K}_{2}\right)\end{array}$ \\
\hline \multirow[t]{3}{*}{9} & FII & $\begin{array}{l}+ \\
+ \\
+ \\
+\end{array}$ & $\begin{array}{l}+ \\
+ \\
+ \\
+\end{array}$ & $\begin{array}{l}- \\
- \\
- \\
+\end{array}$ & $\begin{array}{l}- \\
- \\
- \\
+\end{array}$ & $\begin{array}{l}\text { FII } \\
\text { IgG(K) } \\
\text { IgG (lambda) } \\
\operatorname{IgA}\left(K_{2}\right)\end{array}$ \\
\hline & IgG (lambda) & $\begin{array}{l}+ \\
+ \\
+ \\
+\end{array}$ & $\begin{array}{l}+ \\
+ \\
+\end{array}$ & $\begin{array}{l}+ \\
+ \\
- \\
+\end{array}$ & $\begin{array}{l}+ \\
+ \\
+\end{array}$ & $\begin{array}{l}\text { FII } \\
\operatorname{IgG}(\mathrm{K}) \\
\operatorname{IgG}(\operatorname{lambda}) \\
\operatorname{lgA}\left(\mathrm{K}_{2}\right)\end{array}$ \\
\hline & $\operatorname{IgA}\left(\mathrm{K}_{2}\right)$ & $\begin{array}{l}+ \\
+ \\
+ \\
+\end{array}$ & $\begin{array}{l}+ \\
+ \\
+ \\
-\end{array}$ & $\begin{array}{l}+ \\
+ \\
+ \\
-\end{array}$ & $\begin{array}{l}+ \\
- \\
- \\
-\end{array}$ & $\begin{array}{l}\text { FII } \\
\text { IgG(K) } \\
\text { IgG (lambda) } \\
\operatorname{IgA}\left(\mathrm{K}_{2}\right)\end{array}$ \\
\hline \multirow[t]{2}{*}{7} & $\operatorname{IgG}(\mathrm{K})$ & $\begin{array}{l}+ \\
+ \\
+\end{array}$ & $\begin{array}{l}+ \\
+ \\
+\end{array}$ & $\begin{array}{l}+ \\
+ \\
+\end{array}$ & $\begin{array}{l} \pm \\
\pm \\
-\end{array}$ & $\begin{array}{l}\text { FII } \\
\operatorname{IgG(K)} \\
\operatorname{IgA}\left(K_{2}\right)\end{array}$ \\
\hline & $\operatorname{IgA}\left(\mathrm{K}_{2}\right)$ & $\begin{array}{l}+ \\
+ \\
+\end{array}$ & $\begin{array}{l}+ \\
+ \\
+\end{array}$ & $\begin{array}{l}+ \\
+ \\
+\end{array}$ & $\begin{array}{l}+ \\
+ \\
+\end{array}$ & $\begin{array}{l}\text { FII } \\
\operatorname{IgG}\left(K_{)}\right) \\
\operatorname{IgA}\left(K_{2}\right)\end{array}$ \\
\hline \multirow[t]{2}{*}{3} & $\operatorname{IgG}(\mathrm{K})$ & + & $\begin{array}{l}- \\
- \\
+\end{array}$ & $\begin{array}{l}- \\
\overline{+}\end{array}$ & $\begin{array}{l}- \\
- \\
-\end{array}$ & $\begin{array}{l}\text { FII } \\
\operatorname{IgG}\left(K_{)}\right) \\
\operatorname{IgA}\left(K_{2}\right)\end{array}$ \\
\hline & $\operatorname{Ig} A\left(K_{2}\right)$ & $\begin{array}{l}+ \\
+ \\
+\end{array}$ & $\begin{array}{l}+ \\
+ \\
-\end{array}$ & $\begin{array}{l}+ \\
+ \\
-\end{array}$ & $\begin{array}{l}+ \\
- \\
-\end{array}$ & $\begin{array}{l}\text { FII } \\
\operatorname{IgG}\left(K_{)}\right) \\
\operatorname{IgA}\left(K_{2}\right)\end{array}$ \\
\hline \multirow[t]{2}{*}{10} & $\operatorname{IgG}(\mathrm{K})$ & $\begin{array}{l}+ \\
+ \\
+\end{array}$ & $\begin{array}{l}+ \\
+ \\
+\end{array}$ & $\begin{array}{l}+ \\
- \\
+\end{array}$ & $\frac{+}{+}$ & $\begin{array}{l}\text { FII } \\
\operatorname{IgG(K)} \\
\operatorname{IgA}\left(K_{2}\right)\end{array}$ \\
\hline & $\operatorname{Ig} A\left(\mathrm{~K}_{2}\right)$ & $\begin{array}{l}+ \\
+ \\
+\end{array}$ & $\begin{array}{l}+ \\
+ \\
+\end{array}$ & $\begin{array}{l}+ \\
+ \\
-\end{array}$ & $\begin{array}{l}+ \\
- \\
-\end{array}$ & $\begin{array}{l}\text { FII } \\
\operatorname{IgG(K)} \\
\operatorname{IgA}\left(K_{2}\right)\end{array}$ \\
\hline
\end{tabular}

chest roentgenogram, and pulmonary function studies, this group of patients had remarkably uniform pulmonary disease with I.P.D. and/or chronic or recurrent infections in every patient. Seven of the patients had I.P.D. Of the four patients with no roentgenographically demonstrable I.P.D., two had significantly reduced total vital capacities and hypoxia (1 and 10), with reduced carbon monoxide diffusion capacity in one (1), suggesting that two additional patients may have early interstitial disease. The second common feature of the pulmonary disease was chronic and/or recurrent acute infections documented by culture in seven patients and probable 
Table VII Antibody characterization

\begin{tabular}{|c|c|c|c|c|c|c|}
\hline \multirow[t]{2}{*}{$\begin{array}{l}\text { Patient } \\
\text { No. }\end{array}$} & & \multirow[t]{2}{*}{$\begin{array}{l}\text { Latex } \\
\text { coat }\end{array}$} & \multicolumn{2}{|c|}{$\begin{array}{l}\text { Sucrose } \\
\text { density gradient } \\
\text { Fraction }\end{array}$} & \multicolumn{2}{|c|}{$\begin{array}{l}\text { Sephadex G-200 } \\
\text { Peak }\end{array}$} \\
\hline & & & $19 S$ & $7 S$ & 1 & 2 \\
\hline 7 & IEP* & $\begin{array}{l}\text { IgG } \\
\text { IgA }\end{array}$ & $\begin{array}{l}+ \\
+\end{array}$ & - & $\begin{array}{l}\text { M } \\
+ \\
+\end{array}$ & $\begin{array}{l}\text { G, A } \\
-\end{array}$ \\
\hline 9 & IEP & $\begin{array}{l}\text { IgG } \\
\text { IgA }\end{array}$ & \pm & + & $\begin{array}{l}\mathbf{M} \\
+ \\
-\end{array}$ & $\begin{array}{l}\text { G, A } \\
+ \\
+\end{array}$ \\
\hline 10 & IEP & $\begin{array}{l}\text { IgG } \\
\text { IgA }\end{array}$ & $\stackrel{+}{-}$ & $\bar{t}$ & $\begin{array}{l}\mathbf{M} \\
+ \\
-\end{array}$ & $\begin{array}{l}\text { G, A } \\
\overline{+}\end{array}$ \\
\hline 5 & IEP & $\begin{array}{l}\text { IgG } \\
\text { IgA }\end{array}$ & \pm & $\begin{array}{l}+ \\
+\end{array}$ & $\begin{array}{l}\mathbf{M} \\
+ \\
-\end{array}$ & $\begin{array}{l}\text { G, A } \\
-\end{array}$ \\
\hline 6 & IEP & $\begin{array}{l}\text { IgG } \\
\text { IgA }\end{array}$ & \pm & $\overline{+}$ & $\begin{array}{l}\mathbf{M} \\
+ \\
-\end{array}$ & $\begin{array}{l}\text { G, A } \\
- \\
+\end{array}$ \\
\hline
\end{tabular}

* IEP=immunoelectrophoresis; $\mathrm{G}=\mathrm{IgG} ; \mathrm{A}=\mathrm{IgA}$; $\mathrm{M}=\mathrm{IgM}$.

but without bacteriological confirmation in two patients ( 8 and 6 ). Two patients (5 and 9) did not have infections; both had I.P.D., one associated with rheumatoid arthritis and one with P.I.E. syndrome. The relationship of the P.I.E. syndrome to the I.P.D. is uncertain in Patient 9, but it has been noted that the former may progress to the latter (Carrington, Addington, Goff, Madoff, Marks, Schwaber, and Gaensler, 1969).

The incidence of anti-IgA antibody among patients with rheumatic or immunological disease and associated pulmonary disease is high in this sample of patients. Conversely, the incidence of this antibody among patients with infectious and non-infectious pulmonary disease without associated rheumatic disease is low or absent depending on how one classifies the P.I.E. syndrome, and with the exception of the patient with chronic bronchitis and I.P.D. Anti-IgA antibody was not found in a blood donor population or in hospitalized patients with one exception noted under Results. The anti-IgA antibody was also not found in 52 patients with rheumatic and immunological disease who had no clinically demonstrable pulmonary involvement. These preliminary observations suggest that the anti-IgA antibody may mark a small subgroup of patients with rheumatic and immunological disease that has a diathesis for development of pulmonary disease.

Regarding the separate identity of the anti-IgA and anti-IgG antibodies, the results of the adsorption experiments in all eleven patients support entirely the hypothesis that two separate antibodies or groups of antibodies account for the anti-IgA and anti-IgG activity in these sera. Adsorption of antibody activity from these sera with monoclonal IgA and IgG as immunoadsorbants resulted in selective decrease in, respectively, anti-IgA and anti-IgG titres by two to four tube dilutions. No cross-adsorption was found. Why the adsorption procedure did not decrease antibody titres to zero is uncertain, but certainly not all the insolubilized monoclonal protein which was divided into relatively coarse particles was available for interaction with anti-antibody. Further, insolubilization with glutaraldehyde may not best alter the monoclonal protein to make available the antigenic sites with which these antibodies interact.

The results of the inhibition experiments also support the hypothesis that two separate antibodies account for anti-IgA and anti-IgG activity. In six patients inhibition experiments with FII, monoclonal IgG and monoclonal IgA demonstrated complete separation of antibody activity. In the remaining four patients overlap inhibition (inhibition of FII or IgGcoated latex agglutination by $\operatorname{IgA}$ or the converse) occurred at the highest inhibitor concentration employed. However, it should be noted that the overlap occurred in only one of three experiments in Patient 9 and one of two experiments in Patients 7, 3 , and 10. In the other experiments in these patients complete separation of antibody activity was demonstrated and good separation of antibody activity was also achieved with the adsorption experiments in each of these patients. The reason for the overlap is not known but may represent a nonspecific inhibition effect at the higher protein concentration or measurement of genetic factor or subgroup.

Antibody characterization experiments demonstrated the class of antibody containing the anti-IgA activity to be in the 7S fraction in four of five sera, and also provided further evidence of separation from anti-IgG activity which was in the 19S, IgM fraction only in three of the five sera. We have no information to date relating to the site at which IgA and anti-IgA antibodies interact or to the possible subtype specificities of the anti-IgA antibodies.

A pathogenic relationship between the anti-IgA antibody and the pulmonary disease is speculative. This study was undertaken in part because of the observation that in humans chronic antigenic stimulation may lead to rheumatoid factor formation. In animals there is experimental evidence that antigammaglobulin factors can be produced by administration of altered autologous gammaglobulin (Milgrom and Witebsky, 1960) or autologous gammaglobulin complexed to antigen (Williams and Kunkel, 1963). The observation in this study that anti-IgA antibodies do not occur in patients with primary infectious pulmonary diseases argues against a chronic antigenic stimulus as origin for the anti-IgA antibody. However, there is no way to rule out the 
possibility that the patients with rheumatic diseases and anti-IgA antibody in the serum have a diathesis that allows locally-produced IgA complexed to antigen to be exposed to systemic antibody-producing mechanisms, while patients without this diathesis are able to contain or destroy complexed IgA locally.

It has been postulated that rheumatoid factor as a part of immune complexes may precipitate in vivo in pulmonary capillaries (Tomasi, Fudenberg, and Finby, 1962), possibly leading to inflammation and fibrosis (Cochrane, 1968). Deposition of immunoglobulins in alveolar septa of some patients with idiopathic I.P.D. has been demonstrated (Nagaya, Buckley, and Sieker, 1969). In the absence of biopsy material, there is no direct evidence from patients in this study to support a pathogenic role for immune complexes. However, in three patients, there was clinical evidence (presence of renal disease and lowered serum complement in all and demonstration of glomerular immunoglobulin deposition on renal biopsy in one) of renal immune-complex deposition and one might speculate that such deposition could occur in the pulmonary vasculature as well. The finding of cryoprecipitates in eight of eleven patients in this study is also of interest in this regard. However, there was no clinical evidence of renal disease in the other patients with anti-IgA antibody.

Previous studies have noted antibodies to human IgA in patients with IgA deficiency and patients with transfusion reactions, apparently due to exposure to or administration of incompatible IgA (Fudenberg, Gold, Vyas, and Mackenzie, 1968; Vyas and others, 1969; Ammann and Hong, 1971). In contrast, the patients in this study had not been transfused and had generally high levels of serum IgA; we do not know if they were deficient in an IgA subgroup. Human antibodies to IgA fragments have also been studied but their clinical significance remains to be established (Wilson, Soltis, and Williams, 1970). It is clear from this survey that anti-IgA antibody also occurs in a subgroup of patients with rheumatic diseases, namely those with I.P.D. and chronic or recurrent pulmonary infections. Anti-IgA antibody may eventually provide a unifying concept for pulmonary disease occurring in association with an otherwise apparently heterogenous group of rheumatic diseases.

\section{Summary}

Eleven patients with pulmonary disease, ten of whom had associated rheumatic or immunological disease, had antibodies to IgA in their sera. All also had antiIgG antibody and eight had cryoglobulins. All cryoglobulins contained anti-IgG antibody and five contained anti-IgA antibody. Adsorption and inhibitions experiments demonstrated separation of anti-IgG and anti-IgA activity. Anti-IgA activity was foundō mainly in the $7 \mathrm{~S}$ fraction, and anti-IgG activity, mainly in the $19 \mathrm{~S}$, IgM fraction.

\section{References}

Ammann, A. J., AND Hong, R. (1971) Medicine (Baltimore), 50, 223 (Selective IgA deficiency: presentation of 30 cases and a review of the literature)

Avrameas, S., AND TeRnYNCK, T. (1969) Immunochemistry, 6, 53 (The cross-linking of proteins with glutaraldehyde and its use for the preparation of immunoadsorbants)

BARTfeld, H. (1969) Ann. N.Y. Acad. Sci., 168, 30 (Distribution of rheumatoid factor activity in non-rheumatoid states)

Carrington, C. B., Addington, W. W., Goff, A. M., Madoff, I. M., Marks, A., Schwaber, J. R., and GAENSLER, E. A. (1969) New Engl. J. Med., 280, 787 (Chronic eosinophilic pneumonia)

Cochrane, C. G. (1968) J. Allerg., 42, 113 (The role of immune complexes and complement in tissue injury)

Committee of the American Rheumatism Association (1964) J. Amer. med. Ass., 190, 741 (Primer on the rheumatic diseases, Part IV)

Fudenberg, H. H., Gold, E. R., Vyas, G. N., AND MACKenzie, M. R. (1968) Immunochemistry, 5, 203 (Human antibodies to human IgA globulins)

Hall, J. W., Kozak, M., AND SPINK, W. W. (1964) Amer. J. Med., 36, 135 (Pulmonary infiltrates, pericarditis and eosinophilia)

HenNey, C. S. (1969) Ann. N.Y. Acad. Sci., 168, 52 (Structural and conformational specificity of the antigen for rheumatoid factor)

KeIMOWITZ, R. I. (1964) J. Lab. clin. Med., 63, 54 (Immunoglobulins in normal human tracheobronchial washings)

Mancini, G., Carbonara, A. O., ANd Heremans, J. F. (1965) Immunochemistry, 2, 235 (Immunochemical quantitation of antigens by single radial immunodiffusion)

Milgrom, F., AND Witebsky, E. (1960) J. Amer. med. Ass., 174, 56 (Studies on the rheumatoid and related serum factors I. Autoimmunization of rabbits with gammaglobulin)

NagaYA, H., BuCKLEY, C. E., AND Sieker, H. O. (1969) Ann. intern. Med., 70, 1135 (Positive antinuclear factor in patients with unexplained pulmonary fibrosis)

Pickering, R. J., Gewurz, H., AND Good, R. A. (1968) J. Lab. clin. Med., 72, 298 (Complement inactivation by serum from patients with acute and hypocomplementemic chronic glomerulonephritis) 
Rossen, R. D., Butler, W. T., Waldman, R. H., Alford, R. H., Hornick, R. B., Togo, Y., and Kasel, J. A. (1970) J. Amer. med. Ass., 211, 1157 (The proteins in nasal secretion. II. A longitudinal study of IgA and neutralizing antibody levels in nasal washings from men infected with influenza virus)

Singer, J. M., AND Plotz, C. M. (1956) Ibid., 21, 888 (The latex-fixation test. I. Application to the serologic diagnosis of rheumatoid arthritis)

Smith, C. B., BellaNti, J. A., AND ChANock, R. M. (1967) J. Immunol., 99, 133 (Immunoglobulins in serum and nasal secretions following infection with type 1 parainfluenza virus and injection of inactivated vaccines)

TomasI, T. B. (1968) New Engl. J. Med., 279, 1327 (Human immunoglobulin A)

-, Fudenberg, H. H., AND Findy, N. (1962) Amer.J. Med., 33, 243 (Possible relationship of rheumatoid factors and pulmonary disease)

Vyas, G. N., Holmdahl, L., Perkins, H. A., And Fudenberg, H. H. (1969) Blood, 34, 573 (Serologic specificity of human anti-IgA and its significance in transfusion)

Williams, R. C., AND KUNKel, H. G. (1962) J. clin. Invest., 41, 666 (Rheumatoid factor, complement, and conglutinin aberrations in patients with subacute bacterial endocarditis)

,-- (1963) Proc. Soc. exp. Biol. (N.Y.), 112, 554 (Antibodies to rabbit gammaglobulin after immunizing with various preparations of autologous gammaglobulin)

Wilson, I. D., Soltis, R. D., AND Williams, R. C. (1970) Blood, 36, 390 (Naturally occurring human antibodies to pepsin-digested IgA) 